Kara, Peter A., Tamboli, Roopak R., Cserkaszky, Aron, Martini, Maria G., Barsi, Attila and Bokor, Laszlo (2018) "The perceived quality of light-field video services", Applications of Digital Image Processing XLI., Proceedings of SPIE Volume 10752, ISSN 0277-786X (2018) https://doi.org/10.1117/12.2320562. One print or electronic copy may be made for personal use only. Systematic reproduction and distribution, duplication of any material in this paper for a fee or for commercial purposes, or modification of the content of the paper are prohibited. 


\title{
The perceived quality of light-field video services
}

\author{
Peter A. Kara ${ }^{\mathrm{a}}$, Roopak R. Tamboli ${ }^{\mathrm{b}}$, Aron Cserkaszky ${ }^{\mathrm{b}}$, \\ Maria G. Martini ${ }^{\mathrm{a}}$, Attila Barsi ${ }^{\mathrm{b}}$, and Laszlo Bokor ${ }^{\mathrm{c}}$ \\ ${ }^{a}$ WMN Research Group, Kingston University, Kingston upon Thames, UK \\ ${ }^{\mathrm{b}}$ Holografika, Budapest, Hungary \\ ${ }^{\mathrm{c} B u d a p e s t}$ University of Technology and Economics, Budapest, Hungary
}

\begin{abstract}
Real-time video transmission services are unquestionably dominating the flow of data over the Internet, and their percentage of the global IP packet traffic is still continuously increasing. As novel visualization technologies are emerging, they tend to demand higher bandwidth requirements; they offer more visually, but in order to do so, they need more data to be transmitted. The research and development of the past decades in optical engineering enabled light-field displays to surface and appear in the industry and on the market, and light-field video services are already on the horizon. However, the data volumes of high-quality light-field contents can be immense, creating storing, coding and transmission challenges. If we consider the representation of light-field content as a series of $2 \mathrm{D}$ views, then for a single video frame, angular resolution determines the number of views within the field of view, and spatial resolution defines the 2D size of those views. In this paper, we present the results of an experiment carried out to investigate the perceptual differences between different angular and spatial resolution parametrization of a light-field video service. The study highlights how the two resolution values affect each other regarding perceived quality, and how the combined effects are detected, perceived and experienced by human observers. By achieving an understanding of the related visual phenomena, especially degradations that are unique for light-field visualization, the design and development of resource-efficient light-field video services and applications become more straightforward.
\end{abstract}

Keywords: light-field video, perceived quality, angular resolution, spatial resolution

\section{INTRODUCTION}

As a result of the vast and numerous research efforts addressing light-field visualization, the utilization of this technology in commercial use cases is approaching. Such use cases include, but are not limited to gaming, home multimedia entertainment, teleconferencing/telepresence, public exhibitions and cinema. Different use cases have different visualization requirements. In case of a public exhibition, a wide Field of View (FOV) can be an absolute must in order to support many simultaneous observers, and high angular resolution might be needed if observers viewing the content from a greater distance are also to be visually satisfied. Beyond visualization requirements, other criteria may even be more crucial, such as the total delay in case of real-time applications. It is important to note that most descriptors of light-field systems and services are not independent and affect each other. For example, in case of a light-field video transmission service, higher content spatial and angular resolution can result in higher delay due to the more data to be transmitted. As both contribute to the Quality of Experience (QoE), compromising any of them may result in the critical degradation of user experience. In the scope of this paper, we solely focus on the perceived quality of light-field video, thus delay is not addressed.

Further author information: (Send correspondence to Peter A. Kara)

Peter A. Kara: E-mail: p.kara@kingston.ac.uk

Roopak R. Tamboli: E-mail: r.tamboli@holografika.com

Aron Cserkaszky: E-mail: a.cserkaszky@holografika.com

Maria G. Martini: E-mail: m.martini@kingston.ac.uk

Attila Barsi: E-mail: a.barsi@holografika.com

Laszlo Bokor: E-mail: bokorl@hit.bme.hu 
Visualized on a given light-field display, a video content may have many important quality indicators. If a given video frame is stored as a series of $2 \mathrm{D}$ images before conversion, then content spatial resolution is practically the resolution of those images. Although this is defined by pixels, note that the concept of pixels does not apply once the content is converted and visualized, as light rays hit irregular positions on the screen (holographic diffuser) of the display. Content angular resolution is determined by the ratio of the number of these 2D images and the FOV in which the converted content is shown. As an example, if there are twice as many views as the size of the FOV (given in degrees), then the content angular resolution is 2 views per degree, or 0.5 degree (a view for every 0.5 degree); both descriptions are correct and are used in the scientific community. Content FOV is not addressed often in this context, as it is typically set to match the capability of the display (e.g., rendering light-field content in the FOV of the display). If the content FOV is greater than what the display can support, then the views of the content outside the valid FOV will not be visualized. If the content FOV is the smaller one, then the common practice is that the two sides of the valid FOV without content information will display the given view on the edge (left-end or right-end view) in a 2D manner (the view of the scene or object will not change based on observer location, thus no parallax effect). Therefore, content FOV only affects user experience if it is insufficient for the display system. Content depth can be looked at as a quality indicator as well, as the lack of depth can limit the 3D experience. It is the distance the content perceptually "moves out" from the plane of the screen, either towards the observer or in the other direction. It is bound by the depth budget of the display, which also sets the location of the valid FOV; observers entering the system's utilized depth budget area do not perceive a portion of the visual information (that would be the closest to them, seen from inside the valid FOV), resulting in an invalid perceived light-field. Finally, the frame rate of light-field videos is analogous to $2 \mathrm{D}$ and stereoscopic $3 \mathrm{D}$ videos.

From the aforementioned quality indicators, this paper addresses spatial and angular resolution. The degradation of spatial resolution results in blurred content, instead of an extent of pixelation that is uniform across the screen. Blur in glasses-free 3D visualization is not inherently perceived as something harmful to quality; in fact, it can actually contribute to the visual realism of artificially generated content. It is sufficient to consider the past several decades of research effort regarding spatial anti-aliasing and similar methods. While this already applies to static content, video can introduce additional blur in frames, such as motion blur. Furthermore, motion can actually mask such degradations, either making them unnoticeable or lessens the effect on perceived quality.

Insufficient content angular resolution disturbs the parallax effect. In case of horizontal-parallax-only (HPO) displays, the common terminology is the smooth horizontal motion parallax effect. The words "smooth" and "motion" are sometimes left out, depending on the scientific context. Motion refers to the fact that the true visual experience of the parallax effect requires observer motion within the valid FOV. Of course this does not mean that the parallax effect only applies if the observer is moving. It only means that movement enhances the overall experience of the visual phenomenon simulated by the optical system; similarly to the real-life use case of viewing the world from a moving vehicle, objects close shift their perceived positions faster than those in the distance. Also, observer movement can enable major alterations in perceived occlusions, depending on the visualized scene. Smooth in this context means that as the observer moves, the perceived light-field is virtually continuous and there are no sudden jumps between discrete views. Before continuing on this trail of thought, at this point it is vital to elaborate the statement that no observer motion at all is required for motion parallax. Moreover, the observer does not even need to have two eyes to perceive this effect. Also, the minimal, natural movement of the still human head can be completely discarded. Having one single eye in a fixed position (but not fixed orientation) is sufficient for the parallax effect, as the changing orientation of the eye alone is capable of providing the necessary visual information to the brain. Summa summarum, no matter how the observer views the content from inside the FOV, the parallax effect will always be perceived. That is, unless the content before the conversion does not have a view density that is high enough. Low content angular resolution leads to the crosstalk effect, in which adjacent views interfere with each other. This on its own can be a significant degradation to the perceived quality. However, below given thresholds, not only the smoothness of the horizontal parallax effect becomes compromised, but the entire parallax effect and the visual experience turns corrupted when discrete image borders appear and the content jumps between views. It also comes with a ripple-like visual effect during horizontal observer movement. Yet smaller extents of angular resolution reduction may go unnoticed, and applying such to video content may also have different levels of perceptual toleration compared to static content. 
In this paper, we introduce the results of a subjective quality assessment experiment on light-field video, investigating the perceptual effects of spatial and angular resolution reduction. Test participants were shown video stimuli in pairs, and they were asked to assess the level of perceived degradation (when compared to reference quality) and to choose preferred stimuli (when comparing degraded stimuli), depending on the test type. The aim of the research was to study the perceptual tolerance and the subjective preference regarding the investigated visual phenomena, that may affect user experience.

The reminder of this paper is structured as follows: Section 2 introduces the relevant related research efforts regarding light-field visualization. The experimental setup is presented in Section 3, detailing the test environment, the protocol, the light-field video sequences and the human observer pool. The obtained results are analyzed in Section 4. The paper is concluded in Section 5, highlighting potential future work.

\section{RELATED WORK}

At the time of this paper, there is already a growing literature on light-field QoE, and the spread of commercially available display systems shall further enrich the research efforts within the scientific community. Light-field technology has not yet entered the consumer market, at least on the display side. In recent years, Lytro ${ }^{1}$ has introduced short-baseline light-field cameras, becoming the first to deliver this capture technology to the consumers. However, earlier this year, in 2018, the company announced shutting down*, reverting the presence of light-field technology on the consumer market.

The visualization quality of light-field display systems has been addressed by several researchers. The works of Kovács et al. mainly investigate the spatial ${ }^{2}$ and angular ${ }^{3}$ resolution of such displays, using both subjective tests and optical measurements. ${ }^{4}$ Performing a well-conducted study on perceived quality for this novel visualization technology can prove challenging, due to the lack of standardized testing procedures. Although subjective measurement methodologies have not yet been standardized, the topic has been particularly investigated by the studies of researchers such as Viola et al. ${ }^{5}$ and Darukumalli et al. ${ }^{6}$

The research efforts of Cserkaszky et al. aimed at view synthesis via interpolation, ${ }^{7}$ introduced an angularly continuous display-independent light-field format ${ }^{8}$ and studied the perceived differences between this novel format and the conventional linear camera array visualization. ${ }^{9}$ The works highlight the sensitivity of human observers towards degradations in content angular resolution, and conclude that other visual degradations — such as inaccurately interpolated intermediate views due to insufficient input - can be significantly more tolerated than the disturbances in the smoothness of horizontal parallax.

Tamboli et al. addressed the light-field QoE related to content angular resolution ${ }^{10}$ and proposed an objective $3 \mathrm{D}$ quality metric ${ }^{11}$ based on the findings, applied this metric not only to regular static content but to video frames as well, ${ }^{12}$ investigated the degradations introduced by view synthesis, ${ }^{13}$ studied the connection between basic content features and $\mathrm{QoE}^{14}$ and created a new light-field model data-set with high angular resolution. ${ }^{15}$

The works of Kara et al. focus on content spatial ${ }^{16}$ and angular ${ }^{17}$ resolution, display FOV ${ }^{18}$ the perceived effects of light-field reconstruction ${ }^{19}$ and the viewing conditions of static content. ${ }^{20}$ The findings indicate that reduced spatial resolution can be greatly tolerated, and the difference can be difficult to perceive. The obtained results also show subjective variation in the tolerance towards content angular resolution reduction, but generally high sensitivity can be concluded.

The previously mentioned disseminations of knowledge address passive use cases; content observation without any user interaction. The topic of interaction is investigated by the works of Viola et al. ${ }^{21}$ and Adhikarla et al. ${ }^{22}$ the latter highlighting task performance. In this paper, we study a passive use case, namely video watching.

In a recent work, leading up to this research, the interdependence between content spatial and angular resolution of static models was investigated. ${ }^{23}$ The experiment was conducted in a way to investigate the possible benefits of lowering spatial resolution when angular resolution is already insufficient. The results indicate that such transformation can support the perceived smoothness of the horizontal motion parallax by blurring the disturbances caused by low content angular resolution.

\footnotetext{
*https://www.theverge.com/2018/3/27/17166038/lytro-light-field-camera-company-shuts-down-google-hiring
} 
These findings were used to propose $\mathrm{e}^{24}$ and evaluate ${ }^{25}$ the concept of dynamic adaptive streaming for lightfield video services. The evaluation results are in alignment with the findings obtained from static content; reducing spatial resolution in case of low angular resolution does not degrade the QoE significantly due to the aforementioned compensation. It is important to note that while the evaluation was on quality switching (different content resolution values before and after a given frame), in the experiment presented in this paper, quality parameters were unvarying within a stimulus; they only varied between video stimuli, according to the test conditions. In the following section, we introduce the chosen test conditions, along with all the other relevant attributes and parameters of the experimental setup.

\section{EXPERIMENTAL SETUP}

\subsection{Test Environment}

The subjective tests were carried out in an isolated laboratory environment. During the entire process, the test participants were not exposed to any audiovisual distraction. The lighting condition of the room in which the test participants and the light-field display were located was approximately 20 lux. The video stimuli were presented on the HoloVizio C80 light-field cinema, ${ }^{26}$ which is a front-projection system with a 3 -meter-wide screen. The FOV of the system was calibrated for 45 degrees.

The test participants viewed the screen of the light-field display from a distance of $2.5 \mathrm{H}$, which corresponded to 4.6 meters. As this distance is common practice for studies on perceived quality on this given system, its selection was straightforward. However, the same did not apply to the sideways position of the test participant. For static content, observer movement does not pose a major challenge, as the visualized scene does not alter during the spatial relocation of the test participant. In other words, the test participant can see the exact same model or scene from any given location within the valid FOV, just from a different angle, according to the horizontal parallax effect.

In this context, light-field video at the time of this paper is unexplored territory; in the currently available scientific literature, no study addresses the viewing conditions of light-field video. As taking different viewing conditions into consideration was out of the scope of this experiment, we decided to have near-static observers, which means that only minimal, slow sideways movement was enabled, bound by a meter, resulting in a very limited angle of change. The default viewing position was in line with the center of the screen. We aim to separately address the research questions of the viewing conditions of light-field video in future work.

\subsection{Test Conditions and Protocol}

As our research focused on the spatial and angular resolution of light-field video, these two parameters were the only variables. To each source video content, we applied two settings per parameter, resulting in four initial test conditions. The higher resolution was denoted as "High", and the lower one as "Low".

The subjective test itself was carried out using two methodologies. One was the Degradation Category Rating (DCR), which compared test condition containing "Low" setting to the reference quality (where both settings were "High"). The possible options of the comparison scale were as follows: "Imperceptible" (5), "Perceptible but not annoying" (4), "Slightly annoying" (3), "Annoying" (2) and "Very annoying" (1). With this given scale, test participants could record whether they could perceive a difference between the two video stimuli, and what the level of annoyance was in case they did.

The other type was a paired comparison (PC), to directly compare the stimuli containing "Low" setting(s) with each other. The comparison scale had seven options to differentiate the stimuli, which were as follows: "Much better" $(+3)$, "Better" $(+2)$, "Slightly better" $(+1)$, "Same" $(0)$, "Slightly worse" $(-1)$, "Worse" $(-2)$, "Much worse" (-3).

For both scales, we used the temporally-separated double stimulus method. This means that a participant first watched the two test sequences in the pair, and then selected the amount of degradation compared to the reference stimulus, or selected the preferred video from the pair, depending on the type of scale. The list of investigated test conditions - summarizing the aforementioned cases — is given in Table 1. 
Table 1. The investigated test conditions. $\mathrm{S} 1$ is the first stimulus in the pair and $\mathrm{S} 2$ is the second. AR indicates the setting of angular resolution, and SR refers to spatial resolution.

\begin{tabular}{cccccc} 
Test ID & Test type & S1AR & S1SR & S2AR & S2SR \\
\hline A & DCR & High & High & High & Low \\
\hline B & DCR & High & High & Low & High \\
\hline C & DCR & High & High & Low & Low \\
\hline D & PC & High & Low & Low & High \\
\hline E & PC & High & Low & Low & Low \\
\hline F & PC & Low & High & Low & Low \\
\hline
\end{tabular}

Table 2. High and Low spatial resolution settings of the source contents and their durations.

\begin{tabular}{cccc} 
Source ID & High resolution & Low resolution & Duration \\
\hline Red & $1024 \times 768$ & $640 \times 480$ & $14.4 \mathrm{sec}$ \\
\hline Yellow & $1024 \times 768$ & $800 \times 600$ & $13.6 \mathrm{sec}$ \\
\hline Ivy & $960 \times 540$ & $640 \times 360$ & $10 \mathrm{sec}$ \\
\hline Tesco & $1280 \times 720$ & $640 \times 360$ & $12.5 \mathrm{sec}$ \\
\hline Gears & $1920 \times 1080$ & $640 \times 360$ & $7.2 \mathrm{sec}$ \\
\hline
\end{tabular}

The video stimuli were presented to the test participants in a randomized order, clustered by test type. This means that test participants did not have to switch back and forth between the two subjective assessment tasks, making evaluation more straightforward and focused. The experiment also accommodated the option of providing detailed feedback regarding the perceived differences, but it was not mandatory.

\subsection{Source Video Contents and Test Stimuli}

The experiment involved five different source video contents. Red was a set of columns, separately shifting along the $z$ axis from an initial plane, moving towards the observer in a given pattern, then reverting to the original state. Yellow was a similar video, but the color of the columns varied per side, and the columns rotated as well. These source video contents were provided by Freelusion ${ }^{\dagger}$ to Holografika for research purposes, and the aim of content creation was to utilize the depth budget of the light-field display to a great extent.

Ivy was a short video about a static statue and an ivy plant that continuously, slowly grows around the statue. Both the statue and the plant were highly detailed in structure. Tesco contained multiple intense motions throughout the entire video. Basically a box of milk jumps onto a plank and balances on a cup, while the stage beneath is elevating and rotating. These videos were provided by Post Edison ${ }^{\ddagger}$, particularly created for Holografika, with focus on the different type of motions.

Gears was a set of plain-colored RGB gears, rendered by Holografika. The motion was the fast but uniform rotation of the gears, without variation in speed or direction. Due to the simplicity and repetition of the motion, the video was originally created as a brief loop, and thus the frames were repeated to render the source video for the experiment.

All videos had a constant frame rate of 25 FPS, and used a lossless variation of H.264, individually for each camera view. Although coding and data compression schemes and methods are essential for efficient visualization and affect the perceived quality - which is well-reflected in the works of Viola et al. ${ }^{27}$ and Paudyal et al. ${ }^{28}$ this research topic is out of the scope of this paper, and is thus not addressed.

The High and Low settings of spatial resolution varied per source video content, shown in Table 2, together with video duration. Angular resolution had the same settings for every video: the High setting meant an angular resolution of 0.5 degree $^{19}$ (2 source views per degree), and 1 degree $^{7}$ for Low, based on earlier studies. ${ }^{23}$

\footnotetext{
${ }^{\dagger}$ Freelusion Video Mapping + Dance Company (http://freelusion.com/)

${ }^{\ddagger}$ Post Edison Computer Graphics (http://www.postedison.hu/)
} 

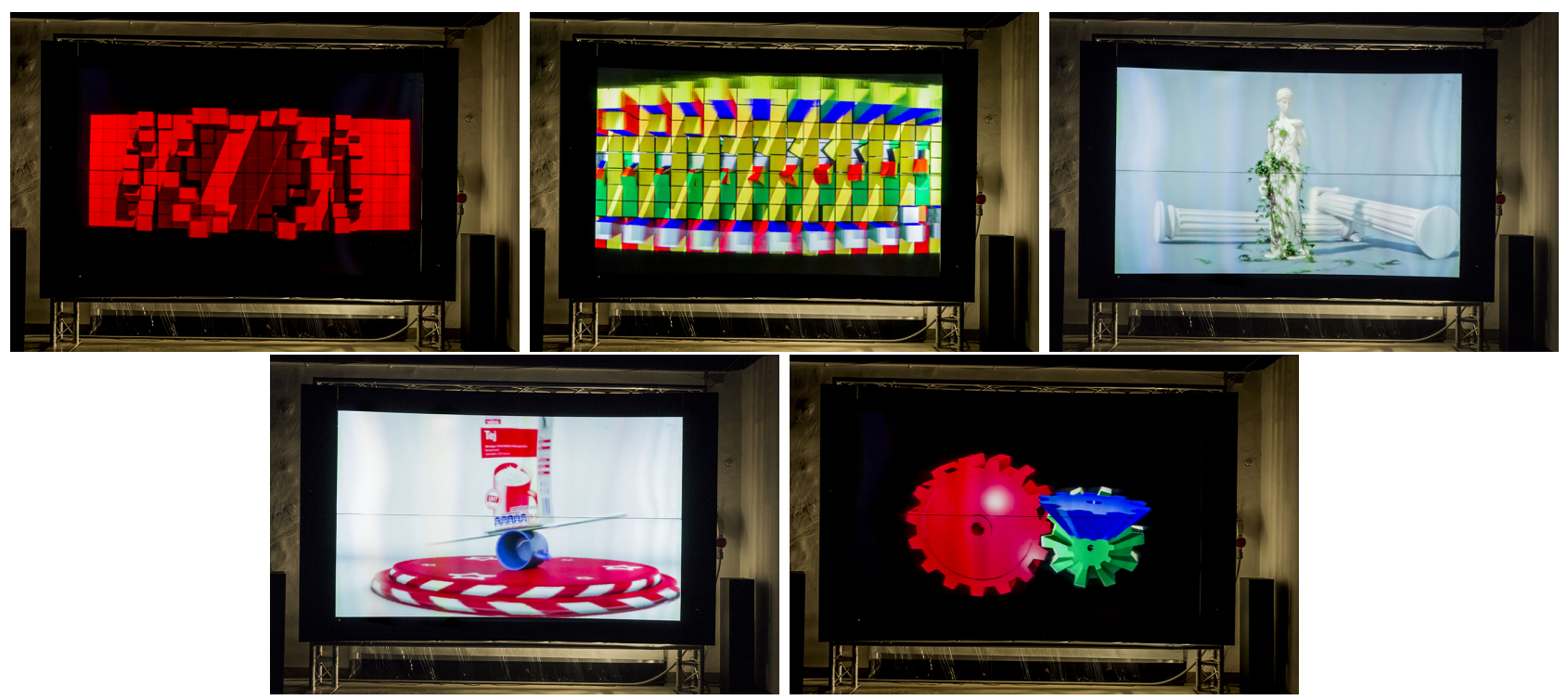

Figure 1. Source video contents (Red, Yellow, Ivy, Tesco and Gears) visualized on the light-field cinema system.

\subsection{Test Participants}

A total of 18 test participants completed the subjective quality experiment. 15 of them were male and 3 were female, from an age range between 21 and 42, with an average age of 28. Before taking part in the test, each test participant was screened for normal vision using the Snellen charts and the Ishihara plates.

\section{RESULTS}

In this section, we introduce the results obtained by the subjective tests. First, we analyze the DCR scores (test $\mathrm{A}, \mathrm{B}$ and $\mathrm{C}$ ), followed by the $\mathrm{PC}$ ratings (test $\mathrm{D}, \mathrm{E}$ and $\mathrm{F}$ ). With 18 test participants and 5 source videos, we collected 90 scores per test, and thus 540 scores in total. Significance in the data analysis was calculated using Tukey HSD, Bonferroni and Holm multiple comparisons.

\subsection{Degradation Category Rating}

Figure 2 introduces the mean results and the distribution of the ratings among the different options. Test A (low spatial resolution) mainly received scores of 4 ("Perceptible but not annoying"), in $58.8 \%$ of the ratings. The mean score of $\mathrm{A}$ was also boosted by the fact that in $27.7 \%$ of the ratings test participants could not detect degradations. Also, test A is the only test for which no score of 2 ("Annoying") and 1 ("Very annoying") was given at all. The mean is above 4 and it is significantly better than what the other two tests obtained.

Although test B (low angular resolution) did receive a higher mean score than test C (low spatial and angular resolution), they are not significantly different from each other. Similarly to test A, no score of 1 was given, however, there were a few scores of 2 ( 8 and 12, respectively for B and $\mathrm{C})$. Generally, while some perceived differences for test B were more without annoyance, it came with slight annoyance for test $\mathrm{C}$.

The important findings here are the relations in significant differences. The p-values of the statistical analysis are shown in Table 3, together with the corresponding significance. The p-values are in alignment with the overlapping confidence intervals of test B and C, although for Holm multiple comparison $p<0.05$ applies. Basically these results indicate that reducing the spatial resolution of the content when content angular resolution is already low will not degrade the perceived quality significantly. In future works, a greater variation in angular resolution for light-field video is targeted, as the compensating blur of low spatial resolution might be perceived differently at even lower source view densities, compared to what was found for static content. ${ }^{23}$ 

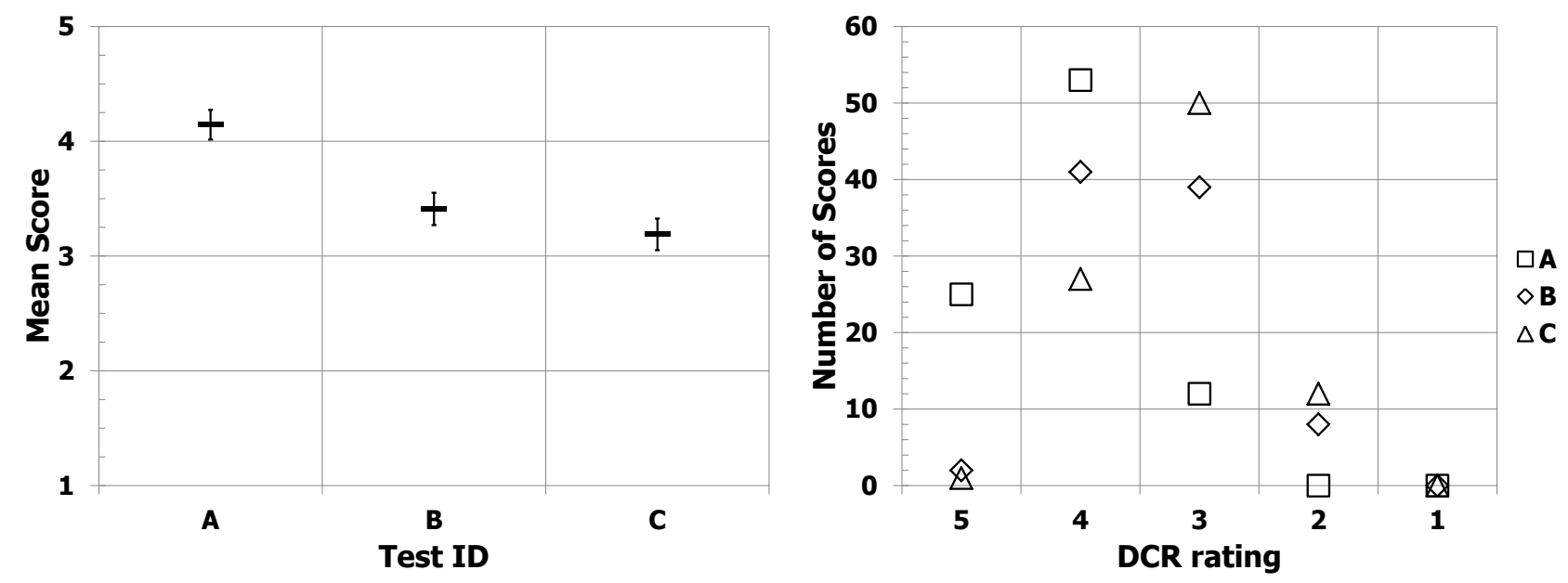

Figure 2. Mean scores with 0.95 CI (left) and rating distribution (right) of the DCR assessment.
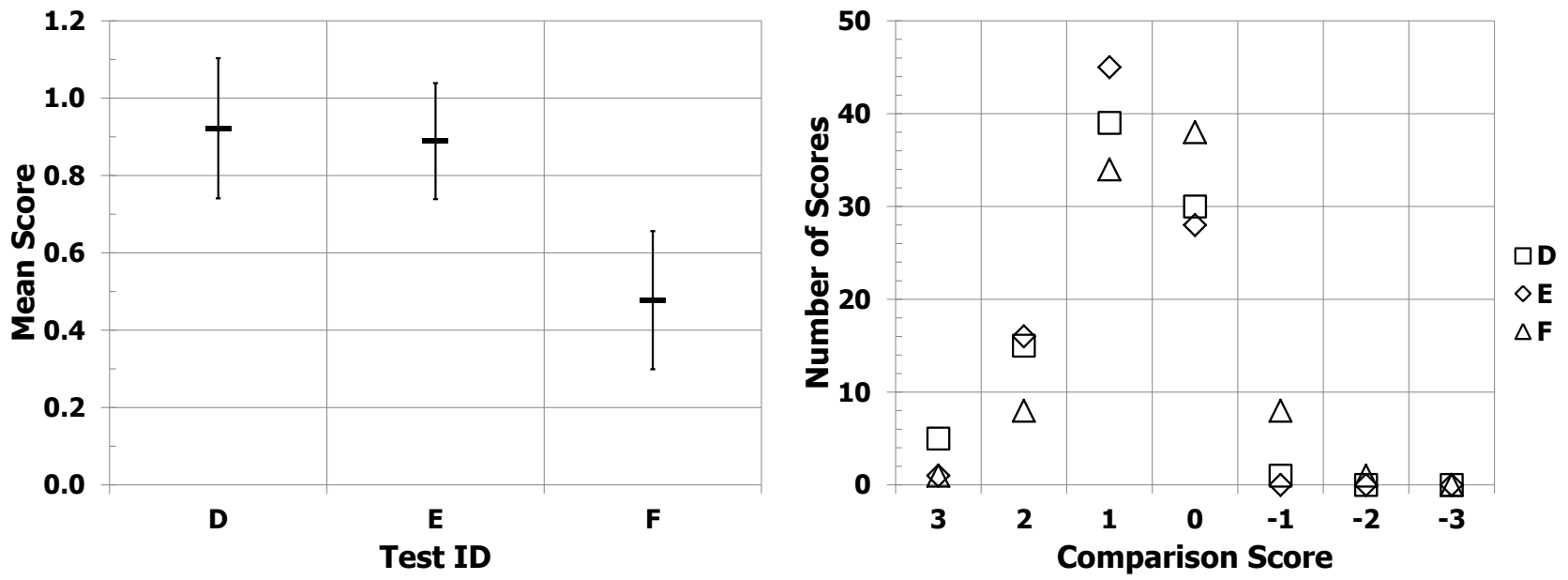

Figure 3. Mean scores with 0.95 CI (left) and rating distribution (right) of the PC assessment.

Regarding the source video contents, the same scoring patterns generally applied. The highest mean scores were obtained by content Tesco and Gears. According to the additional feedback provided by the test participants, the multiple simultaneous motions in content Tesco made it difficult to focus at specific parts of the scene, as attention was divided. In content Gears, this was the opposite, as the main focus was clearly on the edges of the objects, as those were the primary victims of quality degradations. Yet due to this narrowed focus, some perceptual differences went unnoticed. From the 28 "Imperceptible" scores in this part of the experiment, 16 $(57.14 \%)$ were given during the visualization of these two source contents.

This DCR study compared the three types of degraded stimuli to the reference quality. In the following subsection of this paper, the results of the direct comparisons of these test types are introduced and statistically analyzed.

\subsection{Paired Comparison}

In the analysis of the PC scores, positive scores indicate the preference of stimulus 1 (S1) over stimulus 2 (S2), e.g., -1 means that S1 was assessed as "slightly worse" / S2 was assessed as "slightly better". 
Table 3. The p-values of the Tukey HSD (T), Bonferroni (B) and Holm (H) multiple comparisons, and significance (S).

\begin{tabular}{llllll} 
& & $\mathbf{T}$ & $\mathbf{B}$ & $\mathbf{H}$ & $\mathbf{S}$ \\
\hline $\mathrm{A}$ & $\mathrm{B}$ & 0.00 & 0.00 & 0.00 & Yes \\
\hline $\mathrm{A}$ & $\mathrm{C}$ & 0.00 & 0.00 & 0.00 & Yes \\
\hline $\mathrm{B}$ & $\mathrm{C}$ & 0.06 & 0.07 & 0.02 & No \\
\hline $\mathrm{D}$ & $\mathrm{E}$ & 0.89 & 2.35 & 0.78 & No \\
\hline $\mathrm{D}$ & $\mathrm{F}$ & 0.00 & 0.00 & 0.00 & Yes \\
\hline $\mathrm{E}$ & $\mathrm{F}$ & 0.00 & 0.00 & 0.00 & Yes \\
\hline
\end{tabular}

Figure 3 introduces the mean results and the distribution of the ratings among the different options. The first important observation is for test D (low spatial resolution compared to low angular resolution) and $\mathrm{E}$ (low spatial resolution compared to low spatial and angular resolution). As Table 3 indicates as well, the obtained scores are very much alike, and it reinforces the DCR assessments of test B and C. This means that the PC study also shows the lack of significant difference between the stimuli with only angular resolution reduced and the stimuli with both parameters reduced.

As the vast majority of the ratings were either positive or zero, it shows that test participants clearly preferred content spatial resolution reduction over angular resolution reduction, regardless of the change in spatial resolution - as summarized in Table 2. The highest number of 0 ("Same") and -1 ("Slightly worse") scores were used to assess test $\mathrm{F}$, thus in those cases, either the stimuli of test B and C could not be distinguished from each other, or $\mathrm{C}$ was actually perceived to be better. Also, the mean score of test $\mathrm{F}$ is below 0.5 and it is significantly lower than what D and E received. These further support the findings of the DCR test, portraying the lack of the aforementioned significant difference.

As for the difference source video contents, the relations are rather similar to what was seen for the DCR study. Content Tesco and Gears provided the smallest observable differences, and therefore their mean scores are the lowest. Content Red and Yellow received the lowest scores in DCR, and quite similarly the highest scores in PC. This is mostly due to the high utilization of the depth budget of the display. Regarding content Ivy, the scene had the least dynamism with its very subtle animation, yet enabled the test participant to divert more focus to the background and its degradations.

In the optional user feedback, it should be noted that most of the test participants distinguished two types of blur: a general blur (spatial resolution reduction) and a blur that applies more to parts outside the plane of the screen (angular resolution reduction). They both affect the perceived quality of light-field video, but as the obtained results show, angular resolution is more critical.

\section{CONCLUSIONS}

In this paper we presented the results of a subjective quality experiment on the perceived quality of light-field video. Our findings for both DCR and PC tests indicate that the reductions in spatial resolution are significantly more tolerable than in angular resolution. The results also highlight the lack of statistically significant difference between the perceived quality of videos with low angular resolution and with low angular and spatial resolution. On the level of application, this means that if angular resolution is already compromised, reducing spatial resolution will not result in the major degradation of QoE. In future works, we aim to address light-field video viewing conditions, to use real, camera-array-captured content and also to involve longer video sequences, and therefore study QoE over time.

\section{ACKNOWLEDGMENTS}

The work in this paper was funded from the European Union's Horizon 2020 research and innovation program under the Marie Sklodowska-Curie grant agreements No 643072, Network QoE-Net and No 676401, European Training Network on Full Parallax Imaging. 


\section{REFERENCES}

[1] Georgiev, T., Yu, Z., Lumsdaine, A., and Goma, S., "Lytro camera technology: theory, algorithms, performance analysis," in [Multimedia Content and Mobile Devices], 8667, International Society for Optics and Photonics (2013).

[2] Kovács, P. T., Lackner, K., Barsi, A., Balázs, Á., Boev, A., Bregović, R., and Gotchev, A., "Measurement of perceived spatial resolution in 3D light-field displays," in [International Conference on Image Processing (ICIP)], 768-772, IEEE (2014).

[3] Kovács, P. T., Bregović, R., Boev, A., Barsi, A., and Gotchev, A., "Quantifying spatial and angular resolution of light-field 3-D displays," IEEE Journal of Selected Topics in Signal Processing 11(7), 1213$1222(2017)$.

[4] Kovács, P. T., Boev, A., Bregović, R., and Gotchev, A., "Quality measurements of 3D light-field displays," in [Proc. Eighth International Workshop on Video Processing and Quality Metrics for Consumer Electronics (VPQM)], (2014).

[5] Viola, I., Rerabek, M., and Ebrahimi, T., "A new approach to subjectively assess quality of plenoptic content," in [Applications of Digital Image Processing XXXIX], 9971, SPIE (2016).

[6] Darukumalli, S., Kara, P. A., Barsi, A., Martini, M. G., Balogh, T., and Chehaibi, A., "Performance comparison of subjective assessment methodologies for light field displays," in [2016 IEEE International Symposium on Signal Processing and Information Technology (ISSPIT)], 28-33, IEEE (2016).

[7] Cserkaszky, A., Kara, P. A., Barsi, A., and Martini, M. G., "To Interpolate or not to Interpolate: Subjective Assessment of Interpolation Performance on a Light Field Display," in [IEEE International Conference on Multimedia and Expo (ICME) 8th Workshop on Hot Topics in 3D Multimedia (Hot3D)], (2017).

[8] Cserkaszky, A., Kara, P. A., Barsi, A., and Martini, M. G., "Towards display-independent light-field formats," in [2017 International Conference on 3D Immersion (IC3D)], IEEE (2017).

[9] Cserkaszky, A., Kara, P. A., Barsi, A., and Martini, M. G., "Expert Evaluation of a Novel Light-field Visualization Format," in [3DTV Conference], (2018).

[10] Tamboli, R., Vupparaboina, K. K., Ready, J., Jana, S., and Channappayya, S., "A subjective evaluation of true 3D images," in [2014 International Conference on 3D Imaging (IC3D)], IEEE (2014).

[11] Tamboli, R., Appina, B., Channappayya, S., and Jana, S., "Super-multiview content with high angular resolution: 3D quality assessment on horizontal-parallax lightfield display," Signal Processing: Image Communication 47, 42-55 (2016).

[12] Tamboli, R. R., Kara, P. A., Cserkaszky, A., Barsi, A., Martini, M. G., Appina, B., Channappayya, S. S., and Jana, S., "3D Objective Quality Assessment of Light Field Video Frames," in [3DTV Conference], (2018).

[13] Tamboli, R., B., A., Channappayya, S., and Jana, S., "Achieving high angular resolution via view synthesis: quality assessment of 3D content on super multiview lightfield display," in [2017 International Conference on 3D Immersion (IC3D)], IEEE (2017).

[14] Tamboli, R. R., Kara, P. A., Appina, B., Martini, M. G., Channappayya, S. S., and Jana, S., "Effect of Primitive Features of Content on Perceived Quality of Light Field Visualization," in [2018 Tenth International Conference on Quality of Multimedia Experience (QoMEX)], IEEE (2018).

[15] Tamboli, R. R., Reddy, M. S., Kara, P. A., Martini, M. G., Channappayya, S. S., and Jana, S., "A Highangular-resolution Turntable Data-set for Experiments on Light Field Visualization Quality," in [2018 Tenth International Conference on Quality of Multimedia Experience (QoMEX)], IEEE (2018).

[16] Kara, P. A., Kovács, P. T., Martini, M. G., Barsi, A., Lackner, K., and Balogh, T., "Viva la Resolution: The Perceivable Differences between Image Resolutions for Light Field Displays," in [5th ISCA/DEGA Workshop on Perceptual Quality of Systems (PQS 2016)], (2016).

[17] Kara, P. A., Martini, M. G., Kovács, P. T., Imre, S., Barsi, A., Lackner, K., and Balogh, T., "Perceived quality of angular resolution for light field displays and the validity of subjective assessment," in [International Conference on 3D Imaging (IC3D)], (2016).

[18] Kara, P. A., Kovács, P. T., Martini, M. G., Barsi, A., Lackner, K., and Balogh, T., "From a Different Point of View: How the Field of View of Light Field Displays affects the Willingness to Pay and to Use," in [2016 Eighth International Workshop on Quality of Multimedia Experience (QoMEX)], (2016). 
[19] Kara, P. A., Kovács, P. T., Vagharshakyan, S., Martini, M. G., Barsi, A., Balogh, T., Chuchvara, A., and Chehaibi, A., "The Effect of Light Field Reconstruction and Angular Resolution Reduction on the Quality of Experience," in [12th International Conference on Signal Image Technology 83 Internet Based Systems (SITIS) 3rd International Workshop on Quality of Multimedia Services (QUAMUS)], (2016).

[20] Kara, P. A., Cserkaszky, A., Darukumalli, S., Barsi, A., and Martini, M. G., "On the Edge of the Seat: Reduced Angular Resolution of a Light Field Cinema with Fixed Observer Positions," in [9th International Conference on Quality of Multimedia Experience (QoMEX)], (2017).

[21] Viola, I., Rerabek, M., and Ebrahimi, T., "Impact of interactivity on the assessment of quality of experience for light field content," in [9th International Conference on Quality of Multimedia Experience (QoMEX)], (2017).

[22] Adhikarla, V. K., Sodnik, J., Szolgay, P., and Jakus, G., "Exploring direct 3D interaction for full horizontal parallax light field displays using leap motion controller," Sensors 15(4), 8642-8663 (2015).

[23] Kara, P. A., Cserkaszky, A., Barsi, A., Papp, T., Martini, M. G., and Bokor, L., "The Interdependence of Spatial and Angular Resolution in the Quality of Experience of Light Field Visualization," in [2017 International Conference on 3D Immersion (IC3D)], IEEE (2017).

[24] Kara, P. A., Cserkaszky, A., Barsi, A., Martini, M. G., and Balogh, T., "Towards Adaptive Light Field Video Streaming," IEEE COMSOC MMTC Communications - Frontiers , 50-55 (2017).

[25] Kara, P. A., Cserkaszky, A., Martini, M. G., Barsi, A., Laszlo, B., and Balogh, T., "Evaluation of the Concept of Dynamic Adaptive Streaming of Light Field Video," IEEE Transactions on Broadcasting 64(2), 407-421 (2018).

[26] Balogh, T., Nagy, Z., Kovács, P. T., and Adhikarla, V. K., "Natural 3D content on glasses-free light-field 3D cinema," in [ISEST/SPIE Electronic Imaging], International Society for Optics and Photonics (2013).

[27] Viola, I., Rerabek, M., Bruylants, T., Schelkens, P., Pereira, F., and Ebrahimi, T., "Objective and subjective evaluation of light field image compression algorithms," in [Picture Coding Symposium (PCS), 2016], IEEE (2016).

[28] Paudyal, P., Battisti, F., Sjöström, M., Olsson, R., and Carli, M., "Towards the perceptual quality evaluation of compressed light field images," IEEE Transactions on Broadcasting 63(3), 507-522 (2017). 